01

\title{
Радиационные времена жизни уровней энергии палладиеподобных ионов Pr XIV, Nd XV
}

\author{
(C) А.В. Логинов \\ Российский государственный гидрометеорологический университет, \\ 195196 Санкт-Петербург, Россия \\ Петербургский государственный университет путей сообщения, \\ Санкт-Петербург, Россия \\ e-mail: andrlgnv@yandex.ru
}

Поступила в редакцию 15.09.2017 г.

Полуэмпирическим методом промежуточной связи с использованием экспериментальных уровней энергии, известных из литературы, в электродипольном приближении рассчитаны радиационные времена жизни уровней $4 d^{9} 4 f, 4 d^{9} 5 p, 4 d^{9} 5 d, 4 d^{9} 6 s$ в спектрах палладиеподобных ионов $\operatorname{Pr} \mathrm{XIV}, \mathrm{Nd} \mathrm{XV}$. Радиальные интегралы переходов, необходимые для вычисления абсолютных значений вероятностей переходов, получены в форме длины с функциями Хартри-Фока.

DOI: $10.21883 /$ OS.2018.02.45516.204-17

\section{Введение}

Согласно базе данных [1], систематические экспериментальные характеристики спектров палладиеподобных ионов получены вплоть до Nd XV. Для начала этого изоэлектронного ряда (Ag II-In IV) наряду с длинами волн переходов (и полученными из них уровнями энергии) измерены радиационные времена жизни уровней $4 d^{9} 5 p$. Для ионов высоких степеней ионизации значения радиационных констант, известные из литературы, исключительно расчетные, найденные либо полуэмпирически, либо $a b$ initio. В настоящей работе расчет радиационных времен жизни уровней в спектрах палладиеподобных ионов $\mathrm{Pr} \mathrm{XIV}, \mathrm{Nd} \mathrm{XV}$ произведен полуэмпирически, основываясь на экспериментальных значениях уровней энергии из [2]. Рассмотрены электродипольные переходы между четными уровнями $4 d^{10}, 4 d^{9} 5 s, 4 d^{9} 5 d, 4 d^{9} 6 s$ и нечетными $4 d^{9} 4 f, 4 d^{9} 5 p$. Отметим, что настоящий расчет является продолжением работ $[3,4]$, в которых тем же полуэмпирическим методом были рассчитаны вероятности радиационных переходов и времена жизни уровней в палладиеподобных спектрах Ag II-I VIII [3] и Xe IX-Ce XIII [4].

\section{Метод расчета}

Волновые функции промежуточной связи, необходимые для вычисления вероятностей переходов, найдены в одноконфигурационном приближении. Радиальные интегралы, входящие в выражения для матричных элементов оператора энергии, получены методом наименьших квадратов (МНК) по известным [2] уровням энергии. Приняты во внимание электростатическое, спин-орбитальное и так называемое эффективное взаимодействия. Соответствующие величины обозначены в табл. 1-4 как $F_{d l}^{k}, G_{d l}^{k}$ (электростатические интегралы
Слэтера прямого и обменного взаимодействий), $\xi_{4 d}$, $\xi_{n l}$ (спин-орбитальные константы), $F_{k}, G_{k}$ (интегралы Слэтера с запрещенным рангом, „эффективно“ учитывающие вклад двухчастичных взаимодействий, операторы которых действуют только на пространственные координаты), $T_{111}, T_{112}, T_{222}$ (магнитные эффективные параметры, „эффективно“ учитывающие вклад двухчастичных взаимодействий, операторы которых действуют как на пространственные, так и на спиновые координаты). Правила вычисления угловых коэффициентов перед параметрами $F_{d l}^{k}, G_{d l}^{k}, \xi_{4 d}, \xi_{n l}$ общеизвестны (например, [5]), правила вычисления угловых коэффициентов перед эффективными параметрами можно найти в [3]. Здесь только напомним, что для описания межэлектронных взаимодействий в конфигурациях типа $d^{9} l$ (дыркаэлектрон) достаточно привлечь одно- и двухчастичные операторы.

Качество реализации предписания наименьших квадратов определяется дисперсиями параметров, а также стандартными $(\sigma)$ и среднеквадратичными $(\Delta)$ отклонениями по энергии, где

$$
\begin{gathered}
\sigma=\sqrt{\sum_{i=1}^{n}\left(E_{\mathrm{calc}}^{i}-E_{\mathrm{exp}}^{i}\right)^{2} /(n-m)}, \\
\Delta=\sqrt{\sum_{i=1}^{n}\left(E_{\mathrm{calc}}^{i}-E_{\mathrm{exp}}^{i}\right)^{2} / n},
\end{gathered}
$$

$n$ - число экспериментальных уровней, включенных в процедуру МНК, $m$ - число свободно варьируемых параметров, $E_{\text {calc }}^{i}, E_{\text {exp }}^{i}-$ соответственно вычисленное и экспериментальное значения энергии $i$-го уровня.

Из табл. 1-4 видно, что электростатические интегралы $F_{d l}^{k}, G_{d l}^{k}$ и спин-орбитальные константы $\xi_{4 d}, \xi_{n l}$ хорошо определены для всех рассмотренных конфигураций. Сопоставление с соответствующими величинами из $[3,4]$ для Ag II-Ce XIII показывает также, что эти параметры монотонно меняются с изменением заряда 
Таблица 1. Параметры (в $\left.\mathrm{cm}^{-1}\right)$ матрицы энергии конфигураций $4 d^{9} 5 s, 4 d^{9} 6 s$

\begin{tabular}{c|c|c|c}
\hline \multirow{2}{*}{ Параметр } & \multicolumn{2}{|c|}{$4 d^{9} 5 s$} & $4 d^{9} 6 s$ \\
\cline { 2 - 4 } & $\operatorname{Pr}$ XIV & Nd XV & $\operatorname{Pr}$ XIV \\
\hline$F_{d s}^{0}$ & $103556 \pm 33$ & $115152 \pm 34$ & $191368 \pm 5$ \\
$G_{d s}^{2}$ & $21285 \pm 326$ & $22085 \pm 338$ & $6571 \pm 50$ \\
$\xi_{4 d}$ & $12703 \pm 27$ & $14313 \pm 28$ & $12762 \pm 4$ \\
$\sigma$ & 65 & 68 & 10 \\
$\Delta$ & 32 & 34 & 5
\end{tabular}

ядра. Для параметров, описывающих эффективные взаимодействия, ситуация не столь однозначная. Так, в случае уровней $4 d^{9} 5 d$ (табл. 2) из процедуры наименьших квадратов исключены обменные интегралы Слэтера с запрещенным рангом $G_{1}, G_{3}$, хотя для последовательности Xe IX-Ce XIII [4] они были определены вполне приемлемо. То же самое произошло с эффективными параметрами $F_{1}, G_{2}$ (конфигурация $4 d^{9} 5 p$, табл. 4), ранее существенно улучшившими результат подгонки для уровней $4 d^{9} 5 p$ в изоэлектронной последовательности $\mathrm{Ag}$ II-Ce XIII $[3,4]$. Однако в целом учет эффективныX взаимодействий заметно уменьшил параметры $\sigma$ и $\Delta$ (табл. 1-4).

\section{Результаты и обсуждение}

Полученные функции промежуточной связи использованы далее для расчета вероятностей электродипольных переходов. При этом радиальные интегра- лы переходов найдены в форме длины с радиальными функциями, рассчитанными методом Хартри-Фока по программе [6]. Суммированием вероятностей переходов $4 d^{9} 5 p \rightarrow 4 d^{10}, 4 d^{9} 5 p \rightarrow 4 d^{9} 5 s, 4 d^{9} 5 d \rightarrow 4 d^{9} 5 p$, $4 d^{9} 5 d \rightarrow 4 d^{9} 4 f, 4 d^{9} 6 s \rightarrow 4 d^{9} 5 p, 4 d^{9} 4 f \rightarrow 4 d^{10}$ найдены времена жизни уровней $4 d^{9} 4 f, 4 d^{9} 5 p, 4 d^{9} 5 d, 4 d^{9} 6 s$ (табл. 5-8). Для идентификации уровней в этих таблицах принята следующая система обозначений - уровни обозначаются двумя цифрами, первая из которых дает значение полного момента $J$, а вторая - порядковый номер данного уровня среди совокупности уровней с одним и тем же значением $J$ и упорядоченных по возрастанию энергии. В табл. 5 приведены времена жизни уровней $4 d^{9} 4 f$ только с $J=1$, поскольку только с этих уровней возможны электродипольные переходы в нижележащее основное состояние $4 d^{10}(J=0)$, и эти времена жизни есть фактически обратные значения вероятностей переходов с указанных уровней $4 d^{9} 4 f$ на уровень $4 d^{10}$.

Экспериментальные данные для сравнения с содержимым табл. 5-8 в литературе отсутствуют. По этой причине результаты настоящей работы сравниваются исключительно с расчетными данными - из работ [2] (полуэмпирический расчет) и [7] (расчет ab initio). Данные [2,7] выбраны для сравнения из всех представленных в литературе (полную библиографию по вероятностям переходов можно найти в [1]), поскольку их можно непосредственно сопоставить с результатами настоящей работы.

В работе [2] применялся так называемый обобщенный МНК, когда в качестве варьируемых параметров служат не сами радиальные интегралы матрицы энергии, а коэффициенты разложения этих параметров по степе-

Таблица 2. Параметры $\left(\right.$ в $\left.\mathrm{cm}^{-1}\right)$ матрицы энергии конфигурации $4 d^{9} 5 d$

\begin{tabular}{c|c|c|c|c|r}
\hline Параметр & Pr XIV & Nd XV & Параметр & Pr XIV & Nd XV \\
\hline$F_{d d}^{0}$ & $160886 \pm 19$ & $176803 \pm 21$ & $\xi_{5 d}$ & $3549 \pm 23$ & $4144 \pm 21$ \\
$F_{d d}^{2}$ & $46104 \pm 246$ & $49222 \pm 253$ & $F_{1}$ & - & $18 \pm 12$ \\
$F_{d d}^{4}$ & $27791 \pm 412$ & $27671 \pm 409$ & $F_{3}$ & $105 \pm 26$ & $110 \pm 25$ \\
$G_{d d}^{0}$ & $9342 \pm 16$ & $10085 \pm 15$ & $G_{1}$ & - & - \\
$G_{d d}^{2}$ & $12946 \pm 307$ & $14383 \pm 310$ & $G_{3}$ & - & 87 \\
$G_{d d}^{4}$ & $11953 \pm 532$ & $13419 \pm 653$ & $\sigma$ & 65 & 58 \\
$\xi_{4 d}$ & $12736 \pm 20$ & $14305 \pm 19$ & $\Delta$ & &
\end{tabular}

Таблица 3. Параметры $\left(\right.$ в $\left.\mathrm{cm}^{-1}\right)$ матрицы энергии конфигурации $4 d^{9} 4 f$

\begin{tabular}{c|c|c|c|c|c}
\hline Параметр & Pr XIV & Nd XV & Параметр & Pr XIV & Nd XV \\
\hline$F_{d f}^{0}$ & $115818 \pm 53$ & $121659 \pm 84$ & $\xi_{4 f}$ & $1188 \pm 31$ & $1487 \pm 43$ \\
$F_{d f}^{2}$ & $118096 \pm 502$ & $125903 \pm 837$ & $F_{1}$ & $-26 \pm 17$ & $-42 \pm 24$ \\
$F_{d f}^{4}$ & $86094 \pm 758$ & $92616 \pm 1276$ & $F_{3}$ & - & $81 \pm 50$ \\
$G_{d f}^{1}$ & $131681 \pm 100$ & $137508 \pm 134$ & $G_{2}$ & - & - \\
$G_{d f}^{3}$ & $89994 \pm 678$ & $92898 \pm 939$ & $G_{4}$ & $-75 \pm 51$ & $-97 \pm 87$ \\
$G_{d f}^{5}$ & $67034 \pm 1423$ & $69648 \pm 2121$ & $\sigma$ & 155 & 205 \\
$\xi_{4 d}$ & $12329 \pm 48$ & $13877 \pm 66$ & $\Delta$ & 106 & 133
\end{tabular}


Таблица 4. Параметры $\left(\right.$ в $\left.\mathrm{cm}^{-1}\right)$ матрицы энергии конфигурации $4 d^{9} 5 p$

\begin{tabular}{c|c|c}
\hline Параметр & Pr XIV & Nd XV \\
\hline$F_{d p}^{0}$ & $126718 \pm 29$ & $140279 \pm 9$ \\
$F_{d p}^{2}$ & $53394 \pm 513$ & $57688 \pm 148$ \\
$G_{d p}^{1}$ & $15603 \pm 186$ & $18543 \pm 51$ \\
$G_{d p}^{3}$ & $18062 \pm 562$ & $20248 \pm 168$ \\
$\xi_{4 d}$ & $12650 \pm 33$ & $14374 \pm 8$ \\
$\xi_{5 p}$ & $31731 \pm 141$ & $35651 \pm 18$ \\
$F_{1}$ & - & - \\
$G_{2}$ & - & - \\
$T_{111}$ & $4519 \pm 116$ & - \\
$T_{112}$ & $1733 \pm 768$ & $-1364 \pm 128$ \\
$T_{222}$ & $3586 \pm 1561$ & - \\
$\sigma$ & 99 & 30 \\
$\Delta$ & 49 & 19
\end{tabular}

Таблица 5. Времена жизни (в ns) уровней $4 d^{9} 4 f$

\begin{tabular}{c|c|c|c|c}
\hline \multirow{2}{*}{ Уровни } & \multicolumn{2}{|c|}{ Pr XIV } & \multicolumn{2}{c}{$\mathrm{Nd}$ XV } \\
\cline { 2 - 5 } & $\begin{array}{c}\text { настоящая } \\
\text { работа }\end{array}$ & {$[2]$} & $\begin{array}{c}\text { настоящая } \\
\text { работа }\end{array}$ & {$[2]$} \\
\hline 11 & 2.26 & 2.31 & 2.10 & 2.14 \\
12 & 0.197 & 0.200 & 0.162 & 0.158 \\
13 & $3.18 \cdot 10^{-4}$ & $3.15 \cdot 10^{-4}$ & $3.00 \cdot 10^{-4}$ & $2.75 \cdot 10^{-4}$
\end{tabular}

Таблица 6. Времена жизни (в ns) уровней $4 d^{9} 5 p$

\begin{tabular}{c|l|l|c|c|l}
\hline Уровни & Pr XIV & Nd XV & Уровни & Pr XIV & Nd XV \\
\hline 01 & 0.0847 & 0.0729 & 23 & 0.0764 & 0.0658 \\
11 & 0.0300 & 0.0288 & 24 & 0.0686 & 0.0592 \\
12 & 0.00406 & 0.00338 & 31 & 0.150 & 0.135 \\
13 & 0.0168 & 0.0130 & 32 & 0.0677 & 0.0583 \\
21 & 0.155 & 0.139 & 33 & 0.0762 & 0.0655 \\
22 & 0.147 & 0.133 & 41 & 0.0766 & 0.0663
\end{tabular}

Таблица 7. Времена жизни (в ns) уровней $4 d^{9} 5 d$

\begin{tabular}{c|c|l|c|c|c}
\hline Уровни & Pr XIV & Nd XV & Уровни & Pr XIV & Nd XV \\
\hline 01 & 0.0141 & 0.0118 & 24 & 0.0159 & 0.0133 \\
02 & 0.0113 & 0.00968 & 31 & 0.0134 & 0.0111 \\
11 & 0.0141 & 0.0116 & 32 & 0.0161 & 0.0134 \\
12 & 0.0168 & 0.0141 & 33 & 0.0133 & 0.0110 \\
13 & 0.0149 & 0.0123 & 34 & 0.0162 & 0.0134 \\
14 & 0.0152 & 0.0127 & 41 & 0.0135 & 0.0111 \\
21 & 0.0132 & 0.0110 & 42 & 0.0162 & 0.0134 \\
22 & 0.0161 & 0.0133 & 43 & 0.0163 & 0.0135 \\
23 & 0.0135 & 0.0112 & 51 & 0.0160 & 0.0132
\end{tabular}

Таблица 8. Времена жизни (в ns) уровней $4 d^{9} 6 s$ Pr XIV

\begin{tabular}{c|c|c}
\hline Уровни & Настоящая работа & {$[2]$} \\
\hline 11 & 0.0103 & $<0.0106$ \\
21 & 0.0104 & $<0.0093$ \\
22 & 0.0104 & $<0.0091$ \\
31 & 0.0102 & $<0.0088$
\end{tabular}

Таблица 9. Длины волн $(\lambda, \AA)$ и вероятности $\left(A, \mathrm{~s}^{-1}\right)$ переходов $4 d^{9} 5 d \rightarrow 4 d^{9} 5 p$

\begin{tabular}{c|c|c|c|c|c|c|c}
\hline \multirow{2}{*}{ Источник } & \multirow{2}{*}{ Ион } & \multicolumn{2}{|c|}{${ }^{1} S_{0} \rightarrow{ }^{3} P_{1}$} & \multicolumn{2}{c|}{${ }^{1} S_{0} \rightarrow{ }^{1} P_{1}$} & \multicolumn{2}{c}{${ }^{1} S_{0} \rightarrow{ }^{3} D_{1}$} \\
\cline { 3 - 7 } & & $\lambda$ & $A \cdot 10^{-8}$ & $\lambda$ & $A \cdot 10^{-8}$ & $\lambda$ & $A \cdot 10^{-8}$ \\
\hline [7] & Pr XIV & 255 & 144 & 266 & 339 & 288 & 33.4 \\
& Nd XV & 238 & 578 & 250 & 78.4 & 272 & 7.54 \\
Настоящая & Pr XIV & 256 & 181 & 266 & 543 & 289 & 92 \\
работа & Nd XV & 237 & 189 & 249 & 617 & 270 & 114 \\
{$[2]$} & Pr XIV & 256 & 150 & 266 & 530 & - & - \\
& Nd XV & 237 & 250 & 249 & 320 & - & -
\end{tabular}

ням величины $Z_{c}=Z-N_{\mathrm{e}}+1$, где $Z$ - заряд ядра, $N_{\mathrm{e}}$ - число электронов в электронной оболочке ионов изучаемой электронной последовательности (в данном случае $\left.N_{\mathrm{e}}=46\right)$. При этом для реализации предписания наименьших квадратов используются все известные экспериментальные уровни энергии изоэлектронной последовательности в целом. Такой подход основан на априорном предположении о гладкой зависимости радиальных интегралов матрицы энергии от заряда ядра. В наших работах $[3,4]$, включая настоящий расчет, это предположение проверено непосредственно. Вероятности переходов в работе [2] рассчитаны для 107 переходов в спектре Pr XIV и 85 переходов в спектре $\mathrm{Nd}$ XV (т.е. для всех переходов, длины волн которых были измерены в этой работе). В табл. 5 результаты [2] сравниваются с результатами настоящего расчета для времен жизни уровней $4 d^{9} 4 f$. Напомним, что в качестве времен жизни в этой таблице приведены обратные значения вероятностей переходов $4 d^{9} 4 f \rightarrow 4 d^{10}$. В табл. 8 под временами жизни уровней $4 d^{9} 6 s$ иона $\operatorname{Pr} \mathrm{XIV}$ [2] подразумеваются обратные значения сумм вероятностей переходов $4 d^{9} 6 s \rightarrow 4 d^{9} 5 p$, рассчитанных в [2] для каждого из уровней $4 d^{9} 6 s$. Поскольку в цитируемой работе приведены вероятности не для всех разрешенных электродипольных переходов $4 d^{9} 6 s \rightarrow 4 d^{9} 5 p$, постольку времена жизни в колонке [2] табл. 8 следует рассматривать как верхнюю границу этих величин (что и отмечено соответствующим знаком неравенства). Видно, что согласие с результатами работы [2], продемонстрированное в табл. 5, 8, вполне приличное.

В работе [7] использована методика расчета на основе релятивистской теории возмущений с модельным 
потенциалом нулевого приближения. Результаты этого расчета можно сравнить с настоящими результатами, а заодно и с результатами [2], только для вероятностей нескольких переходов $4 d^{9} 5 d \rightarrow 4 d^{9} 5 p$ (табл. 9). Согласие результатов всех трех расчетов не очень впечатляет. Но стоит отметить при этом, что времена жизни, определяемые суммой вероятностей большого числа переходов, менее чувствительны к деталям расчетных методик по сравнению с вероятностями отдельных переходов. В частности, по этой причине в качестве основного результата настоящей работы представлены именно времена жизни, а не вероятности отдельных переходов.

\section{Список литературы}

[1] Kramida A., Ralchenko Yu., Reader J., and NIST ASD Team. NIST Atomic Spectra Database (ver. 5.3). Электронный pecypc. Режим доступа: http://physics.nist.gov/asd

[2] Churilov S.S., Ryabtsev A.N., Wyart J.-F., Tchang-Brillet W.U.L., Joshi Y.N. // Phys. Scr. 2005. V. 71. P. 589.

[3] Логинов А.В., Тучкин В.И. // Опт. и спектр. 1999. Т. 86. № 2. C. 184 .

[4] Логинов А.В. // Опт. и спектр. 2003. Т. 95. № 6. С. 929.

[5] Wybourne B.G. Spectroscopic Properties of the Rare Earths. N.Y.: Wiley, 1965.

[6] Cowan R.D. The Theory of Atomic Structure and Spectra. Berkeley: Univ. Calif. Press, 1981.

[7] Иванова Е.П. // Опт. и спектр. 2003. Т. 94. № 2. С. 181. 